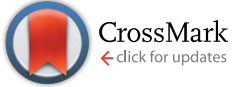

Cite this: RSC Adv., 2016, 6, 82394

Received 11th July 2016

Accepted 25th August 2016

DOI: 10.1039/c6ra17663a

www.rsc.org/advances

\title{
Room temperature ionic liquid assisted synthesis of ultra-stable Au nanoparticles via a modified Brust- Schiffrin method $\uparrow$
}

\author{
Limin Wu, Jingfang Zhou* and Haolan Xu*
}

\begin{abstract}
Au nanoparticles with a small size, narrow size distribution and extraordinary colloidal stability were synthesized via a modified Brust-Schiffrin two phase method. Hydrophobic phosphorus based room temperature ionic liquids (RTIL) were introduced into the toluene phase and solely used as both phasetransfer agent and stabilizer, which significantly simplified the two-phase synthetic system. The synthesized Au nanoparticles are stable for more than two years. Even the thoroughly dried Au NPs can be easily re-dispersed in toluene. Surface characterizations of the Au@RTIL nanoparticles revealed that the amount of the adsorbed RTIL molecules and their degree of order on Au surfaces as well as their interactions with Au surfaces determine the size and stability of the Au@RTIL nanoparticles. The synthetic method and the mechanism presented here are of great importance to the development of the BS method, as well as the Au nanoparticle synthesis and applications.
\end{abstract}

\section{Introduction}

The Au nanoparticle (NP) is one of the most intensively used NPs due to its intrinsic optical, electrical properties, and facile surface modification with bio-functional ligands. ${ }^{1-3}$ These unique physical chemistry properties have driven tremendous applications of Au NPs in catalysis, ${ }^{4-8}$ photoelectronics, ${ }^{2,9,10}$ and biology ${ }^{11,12}$ etc. Synthesis of Au NPs with a wide range of shapes, sizes and surface properties is the prerequisite to pave the way for practical applications and fundamental studies. Up to date, most of Au NPs were synthesized by wet-chemical methods. The solution-based synthesis of Au NPs can be conducted in water and organic solvents. The Turkevich-Frens (TF) and BrustSchiffrin (BS) methods are the two major wet-chemical methods for the synthesis of Au NPs. ${ }^{13,14}$ The TF and its refined methods are the most commonly applied methods to synthesize Au NPs in aqueous phase. However, the obtained Au colloids are generally larger than $10 \mathrm{~nm}$. The NP concentration is relatively low. ${ }^{15,16}$ The BS approach carried out in two-phase system at room temperature can synthesize hydrophobic Au NPs that disperse in oil phase. The synthesized Au NPs generally have smaller sizes $(2-6 \mathrm{~nm})$ and narrow size distribution. ${ }^{15,16}$ The obtained Au NPs can be easily functionalized and modified by subsequent ligand exchange. ${ }^{17,18}$ Recently, it is demonstrated that the BS method has the potential for not only size but also

Future Industries Institute, University of South Australia, Mawson Lakes Campus, SA 5095, Australia.E-mail: jingfang.zhou@unisa.edu.au; haolan.xu@unisa.edu.au $\dagger$ Electronic supplementary information (ESI) available: Digital photographs, TEM images, DLS size distribution, UV-Vis spectra, ToF-SIMs spectra of Au NPs, and table of RTILs. See DOI: 10.1039/c6ra17663a shape controllable Au NP synthesis. ${ }^{19}$ Significantly, the BS method can scale up the production of Au NPs to meet the highly demanded Au applications. ${ }^{16}$

The BS method involves transfer of $\mathrm{AuCl}_{4}{ }^{-}$from water phase to toluene phase, reduction of $\mathrm{Au}(\mathrm{III})$ to $\mathrm{Au}$ metal and stabilization of the generated Au NPs. Typically, tetraoctylammonium bromide (TOAB) is used as phase-transfer agent. However, TOAB is not an excellent stabilizer especially when its concentration is low. Sole utilization of TOAB in BS synthesis can't produce high quality Au NPs. To obtain highly monodisperse and stable Au NPs, additional stabilizer such as alkanethiols which can form strong bond with Au surface is required. ${ }^{14}$ However, the co-existence of TOAB, alkanethiols and $\mathrm{NaBH}_{4}$ in BS phase synthesis has raised ongoing debate regarding the mechanism of the Au nucleation and growth because all these reagents involve in the formation of precursor complexes, reduction, crystal growth and NP stabilization. ${ }^{20-23}$ Therefore, simplifying the BS synthesis and the corresponding growth mechanism is highly required..$^{24,25}$ An ideal strategy is to use only one reagent to simultaneously play the roles of phase transfer agent and stabilizer during the synthesis. Herein, we develop a modified BS method to synthesize Au NPs in twophase without utilization of TOAB and alkanethiols. Hydrophobic room temperature ionic liquids (RTILs) were selected to substitute and play the roles of TOAB and alkanethiols. RTILs are organic salts in liquid state at room temperature. RTILs are recognized as green and environment friendly liquid due to the very low vapour pressure, non-flammability, excellent thermal stability etc. ${ }^{26,27}$ It has been reported that some RTILs such as BMIM- $\mathrm{PF}_{6}$ can efficiently extract $\mathrm{AuCl}_{4}{ }^{-}$from aqueous solution. $^{28}$ Concurrently, some water-soluble RTILs have been 
proven to be novel stabilizers for NPs in aqueous medium. ${ }^{29-31}$ The RTILs can form a robust protective layer to provide both steric and electrostatic repulsion against aggregation of NPs. Inspired by these two merits, we introduce hydrophobic RTILs into toluene phase in BS method to serve both as phase transfer agent and stabilizer for Au NPs synthesis. This strategy renders a relative simple synthetic system and produces high quality $\mathrm{Au}$ NPs.

\section{Experimental}

\section{Chemicals and materials}

Gold(III) chloride trihydrate $\left(\mathrm{HAuCl}_{4}, \geq 99.9 \%\right)$, tetraoctylammonium bromide (TOAB, 98\%), and sodium borohydride $\left(\mathrm{NaBH}_{4}, 99 \%\right)$ were purchased from Sigma-Aldrich (Australia). Toluene was purchased from RCI Labscan (AR). Nitric acid $\left(\mathrm{HNO}_{3}, 70 \%\right)$ and ethanol were purchased from Chem Supply (AR). Hydrochloric acid $(\mathrm{HCl}, 37 \%)$ was purchased from Scharlau. All chemical reagents were used as received without further purification. The water used was purified through a Millipore system. The room temperature ionic liquids (RTILs) were purchased form Io-li-tec ( $>98 \%)$.

\section{Synthesis protocol}

All glassware was cleaned with aqua regia $\left(1: 3\right.$ of $\left.\mathrm{HNO}_{3} / \mathrm{HCl}\right)$ solution and then rinsed thoroughly with Milli-Q water (Caution! Aqua regia solution is dangerous and should be used with extreme care; never store this solution in closed containers.). The gold particles were prepared using a modified BS two-phase method. Take $\mathrm{P}_{14,6,6,6} \mathrm{NTf}_{2}$ (trihexyltetradecylphosphonium bis(trifluoromethylsulfonyl)amide) as example, the detailed synthesis procedure of Au@RTIL NPs is described as below:

Phase transfer of gold salt from aqueous to organic phase. $0.48 \mathrm{mmol}$ of $\mathrm{P}_{14,6,6,6} \mathrm{NTf}_{2}(0.3664 \mathrm{~g})$ was dissolved in $20 \mathrm{~mL}$ of toluene in a round bottom flask. $0.12 \mathrm{mmol}$ of $\mathrm{HAuCl}_{4} \cdot 3 \mathrm{H}_{2} \mathrm{O}$ $(0.04725 \mathrm{~g})$ dissolved in $20 \mathrm{~mL}$ of water was then added. The mixture was stirred vigorously for 30 minutes at room temperature and then kept static for phase separation. The $\mathrm{AuCl}_{4}{ }^{-}$was transferred from the water phase to the toluene phase. The water layer was discarded. The toluene layer contains the transferred gold source, was retained for further use.

Synthesis of Au@P $\mathbf{P}_{14,6,6,6} \mathbf{N T f}_{2}$ NPs. $1.2 \mathrm{mmol}(0.0454 \mathrm{~g})$ of $\mathrm{NaBH}_{4}$ dissolved in $5 \mathrm{~mL}$ of water in a dropping funnel was added dropwise into the toluene phase containing the gold source under stirring over one minute. The reaction solution changed from yellow to wine red. The solution was stirred vigorously for another one hour. After that the aqueous phase was removed.

\section{Characterizations}

UV-Vis spectra of the Au@RTIL NP solutions were recorded with a Shimadzu UV-2600 spectrophotometer by using a $1 \mathrm{~cm}$ quartz cell. The size and size distribution of the Au NPs was measured by dynamic light scattering (DLS) using a Malvern ZetaSizer Nano at a fixed scattering angle of $90^{\circ}$ and at a constant temperature of $25^{\circ} \mathrm{C}$. Transmission electron microscope (TEM) images of the $\mathrm{Au}$ NPs were obtained by a PHILIPS CM100 transmission electron microscope (Tecnai G2 spirit) operated at an accelerating voltage of $100 \mathrm{kV}$. The TEM is equipped with a SIS MegaviewII CCD camera and analySIS image analysis software. The size distributions of the gold nanoparticles were measured using Universal TEM Imaging Platform software and were based on counting at least 200 individual particles. Samples for TEM characterization were prepared by placing a drop of the Au NPs sol on a carbon-coated copper grid followed by drying at room temperature.

Purification of RTIL stabilized Au NPs. The Au NPs stabilized by RTILs, were purified by adding ethanol to remove the free RTIL. $80 \mathrm{~mL}$ ethanol was added to the as-prepared Au@RTIL NPs which dispersed in toluene. Leave it overnight and remove the supernate, the crude product was redissolved in $5 \mathrm{~mL}$ toluene and again precipitated with $50 \mathrm{~mL}$ ethanol.

Fourier transform infrared spectra (FTIR) were determined with a Nicolet 6700 at a resolution of $2 \mathrm{~cm}^{-1}$. The Au@RTIL NPs were deposited on to a $\mathrm{NaCl}$ disc followed by evaporation of toluene solution. Thermal Gravity Analysis (TGA) was performed with an Auto 2950 HR V6.1 A. The temperature range used was from 300 to $873 \mathrm{~K}$.

Time-of-Flight Secondary Ion Mass Spectrometry (ToF-SIMS) experiments were performed using a Physical Electronics Inc. PHI TRIFT V nanoTOF instrument equipped with a pulsed liquid metal ${ }^{79+} \mathrm{Au}$ primary ion gun (LMIG), operating at $30 \mathrm{keV}$ energy. Experiments were performed under a vacuum lower than $5 \times 10^{-6} \mathrm{~Pa}$. "Bunched" $\mathrm{Au}_{1}$ instrumental settings were used to optimise mass resolution for the collection of +SIMS and -SIMS spectra. Surface analyses were performed at a number of locations per sample, typically using a $100 \times 100$ micron raster area. Five locations per sample were analysed to ensure a representative signal from the sample surface. Spectra were interrogated using WincadenceN software (Physical Electronics Inc.).

\section{Results and discussion}

To apply RTILs in BS method, it is required that (1) the RTIL is miscible with toluene but immiscible with water, (2) the RTILs can effectively extract $\mathrm{AuCl}_{4}{ }^{-}$from water phase to toluene phase, (3) RTILs can serve as ligand to stabilize the Au NPs and control the sizes. Sixteen of RTILs were investigated (Table S1 $\dagger$ ) based on rule (1) and rule (2). It is found that a number of RTILs with cation of $\mathrm{P}_{14,6,6,6}$ and anion of $\mathrm{Br}$ met the criteria. 4 of them including $\mathrm{P}_{14,6,6,6} \mathrm{NTf}_{2}, \mathrm{P}_{14,6,6,6} \mathrm{Br}, \mathrm{P}_{14,6,6,6} \mathrm{NH}(\mathrm{CN})_{2}, \mathrm{P}_{14,6,6,6} \mathrm{PF}_{6}$ were selected to study the Au NPs synthesis with the modified BS method (Fig. S1 $\uparrow$ ). Correspondingly, the obtained Au NPs were denoted as Au@RTIL NPs. TEM images of the synthesized Au NPs are shown in Fig. 1. Most of the Au NPs are spherical. The size and size distribution of the NPs depend on the utilized RTILs (Fig. 1 and S2 $†$ ). Fig. 1a shows that the size of the Au@ $\mathrm{P}_{14,6,6,6} \mathrm{NTf}_{2} \mathrm{NPs}$ is $5.8 \pm 2.4 \mathrm{~nm}$, which is close to the value measured by dynamic light scattering (DLS) in Fig. S2. $\dagger$ Similarly, Au NPs with size of $5.7 \pm 1.9 \mathrm{~nm}$ and $5.9 \pm 2.4 \mathrm{~nm}$ are obtained when $\mathrm{P}_{14,6,6,6} \mathrm{Br}$, and $\mathrm{P}_{14,6,6,6} \mathrm{PF}_{6}$ were utilized in the $\mathrm{BS}$ 

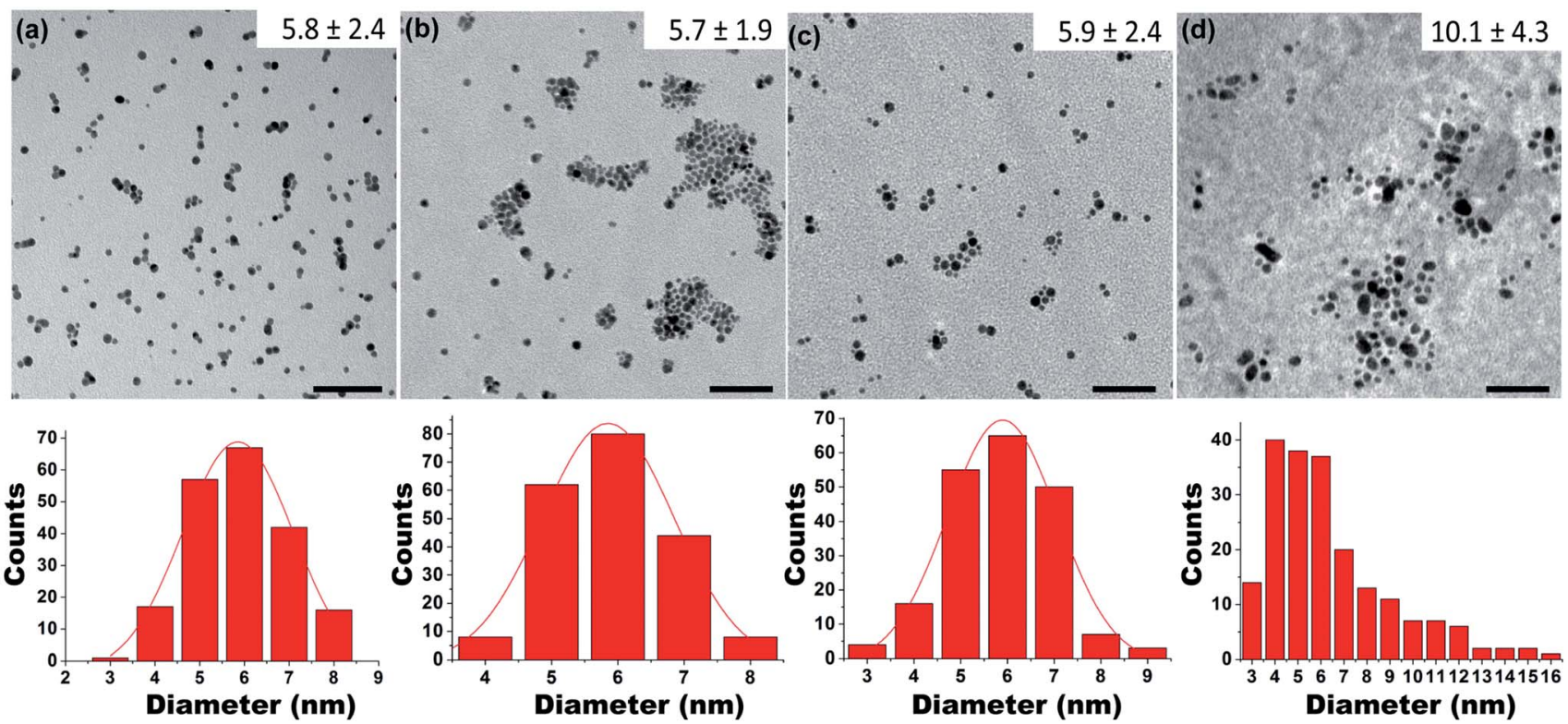

Fig. 1 TEM images and size distribution of (a) Au@P $\mathrm{P}_{14,6,6,6} \mathrm{NTf}_{2}$, (b) $\mathrm{Au}\left(\mathrm{P}_{14,6,6,6} \mathrm{Br}\right.$, (c) $\mathrm{Au}\left(\mathrm{P}_{14,6,6,6} \mathrm{PF}_{6}\right.$, and (d) $\mathrm{Au}\left(\mathrm{P}_{14,6,6,6} \mathrm{NH}(\mathrm{CN})\right)_{2} \mathrm{NPs}$ synthesized by the modified BS method. The molar ratio of $\mathrm{RTIL}$ to $\mathrm{AuCl}_{4}{ }^{-}$is 4 . Scale bar: $50 \mathrm{~nm}$.

synthesis (Fig. $1 \mathrm{~b}$ and c). The $\mathrm{Au} @ \mathrm{P}_{14,6,6,6} \mathrm{NH}(\mathrm{CN})_{2}$ NPs show relatively larger size, wider size distribution $(10.1 \pm 4.3 \mathrm{~nm})$ and nonuniform shapes, which implies that the $\mathrm{P}_{14,6,6,6} \mathrm{NH}(\mathrm{CN})_{2}$ is not excellent ligand for $\mathrm{Au}$ synthesis in BS method (Fig. 1d). However, if the RTILs were substituted by TOAB, rather poor quality Au NPs were obtained (Fig. S3†). These results confirm the feasibility of utilization of the specific hydrophobic RTILs both as phase transfer agent and stabilizer to synthesize high quality $\mathrm{Au}$ NPs by a modified BS method. It is worth noting that in this research the concentration of RTIL/TOAB $(0.024 \mathrm{M})$ is much lower than the concentration of TOAB $(0.05 \mathrm{M})$ in typical BS method. ${ }^{14}$

The size of the Au@RTIL NPs can be controlled by simply adjusting the amount of RTILs. For example, TEM images of the $\mathrm{Au} @ \mathrm{P}_{14,6,6,6} \mathrm{NTf}_{2}$ NPs clearly demonstrated the effect of the molar ratio of $\mathrm{P}_{14,6,6,6} \mathrm{NTf}_{2}$ to $\mathrm{AuCl}_{4}{ }^{-}(R)$ on particle sizes (in this research, the concentration of $\mathrm{AuCl}_{4}{ }^{-}$is fixed). When $R$ is 1 , the diameter of the Au NPs is measured to be $8.8 \pm 2.9 \mathrm{~nm}$ (Fig. 2a). When $R$ is increase to 2,4 , and 8 , the diameter of the Au NPs decreases to $6.5 \pm 2.2 \mathrm{~nm}$ (Fig. 2b), $5.8 \pm 2.4 \mathrm{~nm}$ (Fig. 1a), and $3.9 \pm 1.7 \mathrm{~nm}$ (Fig. $2 \mathrm{c}$ ) respectively. Correspondingly, the UV-Vis spectra of these Au NPs also show noticeable peak shifting (Fig. 2d). The plasmon absorption bands of Au@ $\mathrm{P}_{14,6,6,6} \mathrm{NTf}_{2}$ NPs locate at 518, 517, 515 and $506 \mathrm{~nm}$ when $R$ is $1,2,4$ and 8 respectively, indicating the particle size become smaller with the increase of $\mathrm{P}_{14,6,6,6} \mathrm{NTf}_{2}$. The increase of $\mathrm{P}_{14,6,6,6} \mathrm{NTf}_{2}$ provides more ligands to stabilize Au surfaces which allows the formation of smaller particles with larger overall surface area. However, it is found that further increase of $R$ to 16 induced little change in particle size (Fig. S4†), indicating the saturation of RTIL for Au synthesis.

The colloidal stability of the obtained Au@RTILs NPs was investigated by monitoring the plasmon absorption change of the Au NPs solutions along with the storage time. The original plasmon absorption spectra of the Au@RTIL NPs are shown in Fig. $S 5, \uparrow$ from which one can see the narrow absorption peaks at around $517-520 \mathrm{~nm}$ for $\mathrm{Au} @ \mathrm{P}_{14,6,6,6} \mathrm{NTf}_{2}, \mathrm{Au} @ \mathrm{P}_{14,6,6,6} \mathrm{Br}$, and $\mathrm{Au} @ \mathrm{P}_{14,6,6,6} \mathrm{PF}_{6} \mathrm{NPs}$, indicating the small particle sizes (5-10 $\mathrm{nm}$ ) and narrow size distribution of Au NPs. These results agree well with the size measured by TEM and DLS. The $\mathrm{Au} @ \mathrm{P}_{14,6,6,6} \mathrm{NH}(\mathrm{CN})_{2}$ NPs shows a wide absorption peak, confirming the wide size distribution. When these Au NPs were stored for 30 days, slight change in the absorption intensity and
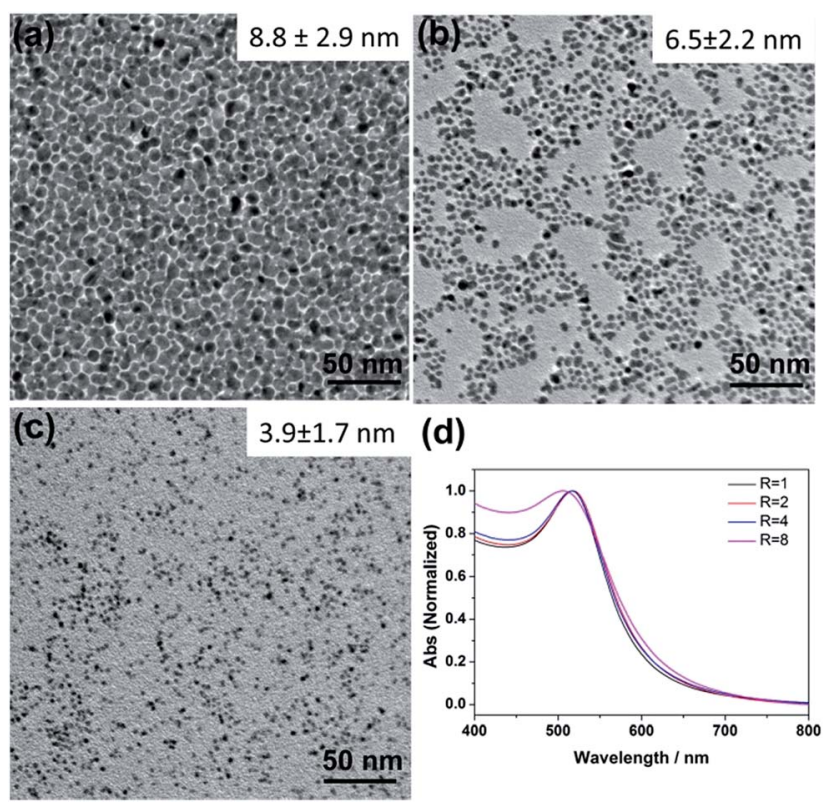

Fig. 2 TEM images of the Au@ $\mathrm{P}_{14,6,6,6} \mathrm{NTf}_{2}$ NPs synthesized when $R$ is (a) 1, (b) 2, (c) 8, and (d) their UV-Vis spectra. 
peak position were observed for the $\mathrm{Au} @ \mathrm{P}_{14,6,6,6} \mathrm{NTf}_{2}$, $\mathrm{Au} @ \mathrm{P}_{14,6,6,6} \mathrm{Br}$, and $\mathrm{Au} @ \mathrm{P}_{14,6,6,6} \mathrm{PF}_{6}$ NPs (Fig. 3a). As comparison the Au NPs synthesized with sole TOAB shows rather poor colloidal stability. The Au@TOAB NPs aggregated and precipitated out quickly within 2 days (Fig. S6†). It is worth noting that the Au@P ${ }_{14,6,6,6} \mathrm{NTf}_{2}$ NPs are still stable even after 2 years storage (Fig. 3b). The toluene was thoroughly evaporated after such a long period of storage, leaving behind the dried $\mathrm{Au} @ \mathrm{P}_{14,6,6,6} \mathrm{NTf}_{2}$ NPs. However, when toluene was added into the vial, the dried $\mathrm{Au}$ NPs were readily re-dispersed in the toluene. No obvious aggregation or precipitation of NPs was observed. This outstanding colloidal stability and redispersibility of Au@RTILs NPs should be originated from the superior stability, non-volatility and liquid state of the adsorbed RTILs on the Au surfaces.

To verify the presence of RTILs on Au NPs surfaces and investigate their roles in stabilization, TGA, FTIR, and ToF-SIMS analysis were conducted. As shown in Fig. 4, TGA measurement clearly depicts the weight loss of all the Au@RTIL NPs. The $\mathrm{Au} @ \mathrm{P}_{14,6,6,6} \mathrm{NTf}_{2} \mathrm{NPs}$ (black curve) exhibits a weight loss $(\Delta W)$ of about $7.72 \%$. Accordingly, the number of RTIL molecules $(N)$ on each Au NPs can be roughly estimated as:
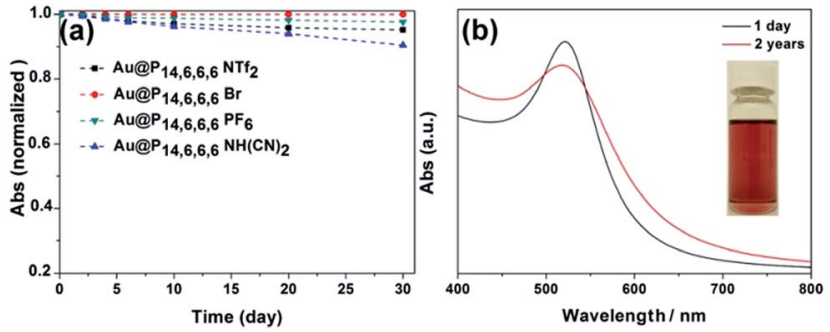

Fig. 3 (a) Plots of the normalized absorbance of Au@RTILs NPs as a function of time; (b) UV-Vis spectra of Au $\mathrm{aP}_{14,6,6,6} \mathrm{NTf}_{2} \mathrm{NP}$ solution stored for 1 day and 2 years, the inset is a photo of Au NP solution after 2 years storage.

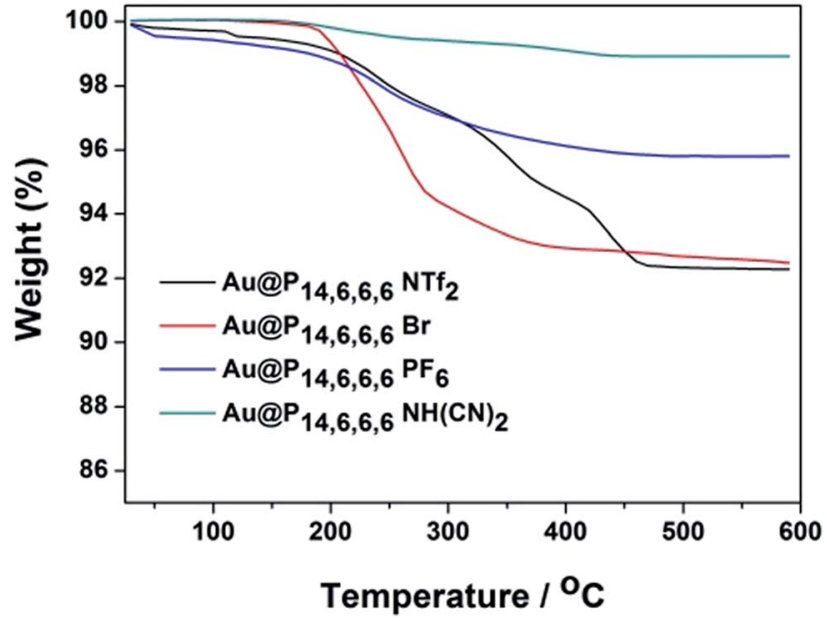

Fig. 4 TGA curves of the Au@P ${ }_{14,6,6,6} \mathrm{NTf}_{2}$, Au@P $14,6,6,6 \mathrm{Br}$, Au@P ${ }_{14,6,6,6} \mathrm{PF}_{6}$ and $A u\left(\mathrm{P}_{14,6,6,6} \mathrm{NH}(\mathrm{CN})_{2} \mathrm{NPs}\right.$.

$$
N=\frac{4 \pi r^{3} \rho N_{\mathrm{A}} \Delta W}{3(1-\Delta W) M_{\mathrm{w}}}
$$

where $r$ is the radius of Au NPs, $N_{\mathrm{A}}$ is Avogadro constant, $M_{\mathrm{w}}$ is molar mass of the RTIL, $\rho$ is the density of the gold ( $\rho=19.3$ $\mathrm{g} \mathrm{cm}^{-3}$ ). The number of $\mathrm{P}_{14,6,6,6} \mathrm{NTf}_{2}$ molecules on each Au NPs is calculated to be about 142 , and the molecular density on unit surface is about $1.26 \mathrm{~nm}^{-2}$. The weight loss of $\mathrm{Au} @ \mathrm{P}_{14,6,6,6} \mathrm{Br}$, $\mathrm{Au} @ \mathrm{P}_{14,6,6,6} \mathrm{PF}_{6}$ and $\mathrm{Au} @ \mathrm{P}_{14,6,6,6} \mathrm{NH}(\mathrm{CN})_{2} \mathrm{NPs}$ are $7.55 \%, 4.81 \%$ and $1.09 \%$ separately. The corresponding RTIL molecular density on unit Au surface is about $1.62 / \mathrm{nm}^{2}, 0.83 / \mathrm{nm}^{2}$, and $0.36 / \mathrm{nm}^{2}$ respectively. It is noticed that the compared with the $\mathrm{P}_{14,6,6,6} \mathrm{NTf}_{2} \mathrm{Au} @ \mathrm{P}_{14,6,6,6} \mathrm{Br}$, and $\mathrm{Au} @ \mathrm{P}_{14,6,6,6} \mathrm{PF}_{6} \mathrm{NPs}$, the $\mathrm{Au} @ \mathrm{P}_{14,6,6,6} \mathrm{NH}(\mathrm{CN})_{2}$ NPs have the lowest RTIL molecular density on the surface, which may response to their relative poor stability.

To further investigate the adsorption of RTILs and their degree of order on Au NP surfaces, FTIR spectra of the Au@RTIL NPs and the corresponding pure RTIL molecules were recorded and compared. As shown in Fig. 5, pure $\mathrm{P}_{14,6,6,6} \mathrm{NTf}_{2}$ shows typical FTIR spectra which is the same with reported data. ${ }^{32}$ The symmetric and asymmetric stretching vibrations of $\mathrm{C}-\mathrm{H}$ of methylene of $\mathrm{P}_{14,6,6,6} \mathrm{NTf}_{2}$ are located at 2856 and $2928 \mathrm{~cm}^{-1}$ respectively. In the low frequency region, peaks are observed at $1468 \mathrm{~cm}^{-1}\left(-\mathrm{CH}_{2}-\right.$ asymmetric bending); $1351 \mathrm{~cm}^{-1}(\mathrm{~S}=\mathrm{O}$ stretching); $1193 \mathrm{~cm}^{-1}, 1138 \mathrm{~cm}^{-1}\left(-\mathrm{CF}_{3}\right) ; 1058.22 \mathrm{~cm}^{-1}(\mathrm{~S}=\mathrm{O}$ stretching). The FTIR spectrum of Au@P $\mathrm{P}_{14,6,6,6} \mathrm{NTf}_{2}$ NPs exhibits methylene stretching modes with slight lower wave numbers at 2849 and $2917 \mathrm{~cm}^{-1}$, indicating highly ordered alkyl chains on the surface of Au NPs. A band splitting of $1468 \mathrm{~cm}^{-1}$ into two peaks positions at 1471 and $1461 \mathrm{~cm}^{-1}$ was observed, indicating the orthorhombic subcell packing of chains with small defects. ${ }^{33}$ Judging together with the TGA result (high RTIL cover density on Au NPs surfaces), it is reasonable to propose that the $\mathrm{Au}$ NPs are densely surrounded by $\mathrm{P}_{14,6,6,6} \mathrm{NTf}_{2}$ shells, which provides strong steric hindrance thus rendering the Au NPs excellent stability. The FTIR spectra of Au@ $\mathrm{P}_{14,6,6,6} \mathrm{Br}$ (Fig. S7a $\dagger$ ), and Au@ $\mathrm{P}_{14,6,6,6} \mathrm{PF}_{6}$ NPs (Fig. S7b $\dagger$ ) all show the

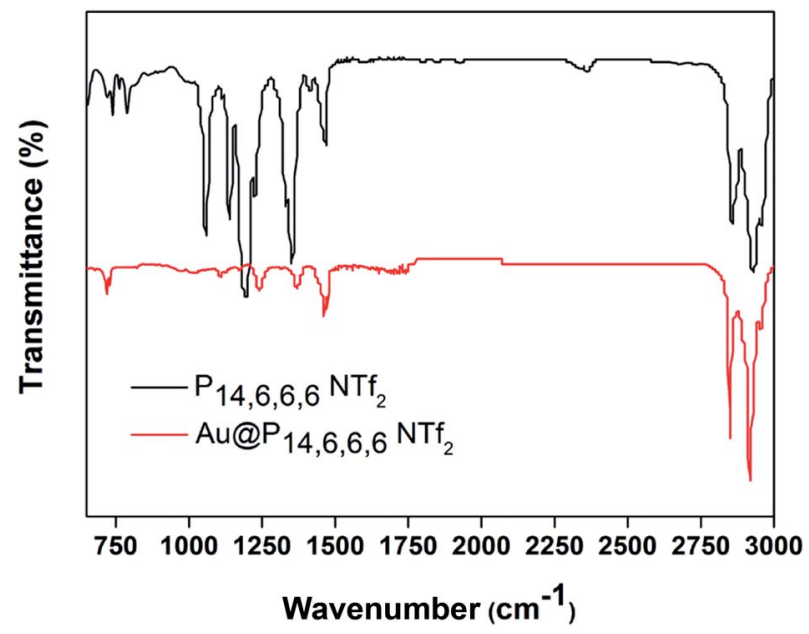

Fig. 5 FTIR spectra of pure $P_{14,6,6,6} \mathrm{NTf}_{2}$ (black curve) and Au@P ${ }_{14,6,6,6} \mathrm{NTf}_{2}$ NPs (red curve). 
characteristic peaks of the corresponding RTILs, confirming the presence of RTILs on the surface of Au NPs. Slight shift of methylene stretching modes to lower wave numbers are also observed in the $\mathrm{Au} @ \mathrm{P}_{14,6,6,6} \mathrm{Br}$ and $\mathrm{Au} @ \mathrm{P}_{14,6,6,6} \mathrm{PF}_{6} \mathrm{NPs}$, indicating the ordered alkyl chains on Au surfaces. Similarly, these orderly arranged RTIL molecules lead to the excellent stability of the Au NPs. In contrast, it is noticed that the peaks of $\mathrm{Au} @ \mathrm{P}_{14,6,6,6} \mathrm{NH}(\mathrm{CN})_{2}$ NPs are highly identical with that of the pure $\mathrm{P}_{14,6,6,6} \mathrm{NH}(\mathrm{CN})_{2}$ (Fig. S7c $\dagger$ ), which implies the weak interactions between the $\mathrm{Au}$ NPs and $\mathrm{P}_{14,6,6,6} \mathrm{NH}(\mathrm{CN})_{2}$, and the loose packing of the alkyl chains on Au NPs. This agrees well with the low RTIL molecular cover density derived from the TGA measurement, explaining the relatively poor quality of the $\mathrm{Au}$ NPs synthesized in the presence of $\mathrm{P}_{14,6,6,6} \mathrm{NH}(\mathrm{CN})_{2}$.

ToF-SIMS is a surface-sensitive method that provides elemental, chemical state, chemical fragment and molecular information from the solid surfaces. The average depth of analysis is approximately $1 \mathrm{~nm}$. Herein the ToF-SIMS spectra of $\mathrm{Au} @ \mathrm{P}_{14,6,6,6} \mathrm{NTf}_{2}, \mathrm{Au} @ \mathrm{P}_{14,6,6,6} \mathrm{Br}$, and $\mathrm{Au} @ \mathrm{P}_{14,6,6,6} \mathrm{PF}_{6}$ NPs were recorded to investigate the possible chemical interactions between the Au surface and RTILs molecules. Fig. 6 is the ToFSIMS spectrum of $\mathrm{Au} @ \mathrm{P}_{14,6,6,6} \mathrm{NTf}_{2}$ NPs which reveals the presence of $\mathrm{CH}^{-}(m / z=13), \mathrm{O}^{-}(m / z=16) ; \mathrm{C}_{2} \mathrm{H}_{5}{ }^{+}(m / z=29) ; \mathrm{Cl}^{-}$ $(m / z=35,37) ; \mathrm{Au}^{-}(m / z=197) ; \mathrm{Au}^{+} \mathrm{Cl}^{-}$and $\mathrm{AuCl}^{2-}(m / z=232$, 267) and $\mathrm{C}_{n} \mathrm{H}_{2 n+2} \mathrm{P}(\mathrm{m} / z=397-485)$. The detected fragments of $\mathrm{Au}^{+} \mathrm{Cl}^{-}$and $\mathrm{AuCl}^{2-}$ confirm the strong chemical interaction/ bond between $\mathrm{Au}$ and $\mathrm{Cl}$. The $\mathrm{Cl}$ is from the precursor $\mathrm{AuCl}_{4}{ }^{-}$ but not the anion of RTIL, because the $\mathrm{P}_{14,6,6,6} \mathrm{NTf}_{2}$ does not contain any $\mathrm{Cl}$. The fragment $\mathrm{C}_{n} \mathrm{H}_{2 n+2} \mathrm{P}$ is from the cation $\left(\mathrm{P}_{14,6,6,6}\right)$ of the RTIL. Therefore, it is safe to propose that in the $\mathrm{Au} @ \mathrm{P}_{14,6,6,6} \mathrm{NTf}_{2} \mathrm{NPs}$, the anion $\mathrm{Cl}^{-}$directly bonds to the $\mathrm{Au}$ surface and the cation of the RTIL $\left(\mathrm{P}_{14,6,6,6}\right)$ with long carbon chain orderly arranges in the outer layer which produce a robust steric hindrance to stabilize the Au NPs. The ToF-SIMS spectrum of $\mathrm{Au} @ \mathrm{P}_{14,6,6,6} \mathrm{PF}_{6}$ NPs (Fig. S8 $\dagger$ ) also clearly show the fragments of $\mathrm{Au}^{+} \mathrm{Cl}^{-}, \mathrm{AuCl}^{2-}$, and $\mathrm{C}_{n} \mathrm{H}_{2 n+2} \mathrm{P}\left(\mathrm{M}^{+}\right)$from cation $\mathrm{P}_{14,6,6,6}$, indicating the similar surface layer structure with that of Au@P $P_{14,6,6,6} \mathrm{NTf}_{2}$ NPs. However, the ToF-SIMS spectrum of $\mathrm{Au} @ \mathrm{P}_{14,6,6,6} \mathrm{Br}$ NPs presents no fragments of $\mathrm{Au}^{+} \mathrm{Cl}^{-}$and $\mathrm{AuCl}^{2-}$, but the fragments of $\mathrm{AuBrH}^{-}, \mathrm{AuBrH}_{2}{ }^{-}, \mathrm{AuBrH}_{3}{ }^{-}(\mathrm{m} / \mathrm{z}$ = 276-279); $\mathrm{C}_{2} \mathrm{H}_{2} \mathrm{AuBr}^{-}, \mathrm{C}_{2} \mathrm{H}_{4} \mathrm{AuBr}^{-}(\mathrm{m} / z=304), \mathrm{C}_{5} \mathrm{H}_{2} \mathrm{AuBr}^{-}$, $\mathrm{C}_{5} \mathrm{H}_{4} \mathrm{AuBr}^{-}(\mathrm{m} / \mathrm{z}=338,340)$ (Fig. S9†). This confirms the existence of large amount of $\mathrm{AuBr}^{-}$fragments, indicating the strong chemical interaction/bond between $\mathrm{Br}$ and $\mathrm{Au}$ surfaces. The $\mathrm{Br}$ is from the anion of $\mathrm{P}_{14,6,6,6} \mathrm{Br}$. The peak of $\mathrm{C}_{n} \mathrm{H}_{2 n+2} \mathrm{P}\left(\mathrm{M}^{+}\right)$from the cation of $\mathrm{P}_{14,6,6,6} \mathrm{Br}$ is also detected. According to these surface characterizations, it is clear that Au has strong chemical interaction with halide ions. As reported, there are unoccupied molecular orbital on the surface of the noble metal NPs. Halide ions with outermost lone pair electrons are strongly adsorbed on the Au surface creating a negatively charged layer. ${ }^{34}$ When the anion of RTIL is $\mathrm{Br}^{-}$, it directly binds to Au surfaces. The cation of RTIL orderly arranges in the outer layer. Therefore, both the cation and anion of RTIL involve in stabilizing Au NPs, leading to the outstanding stability. When the anion of RTIL is not $\mathrm{Br}^{-}$, the $\mathrm{Cl}^{-}$from the $\mathrm{AuCl}_{4}^{-}$strongly binds to the $\mathrm{Au}$

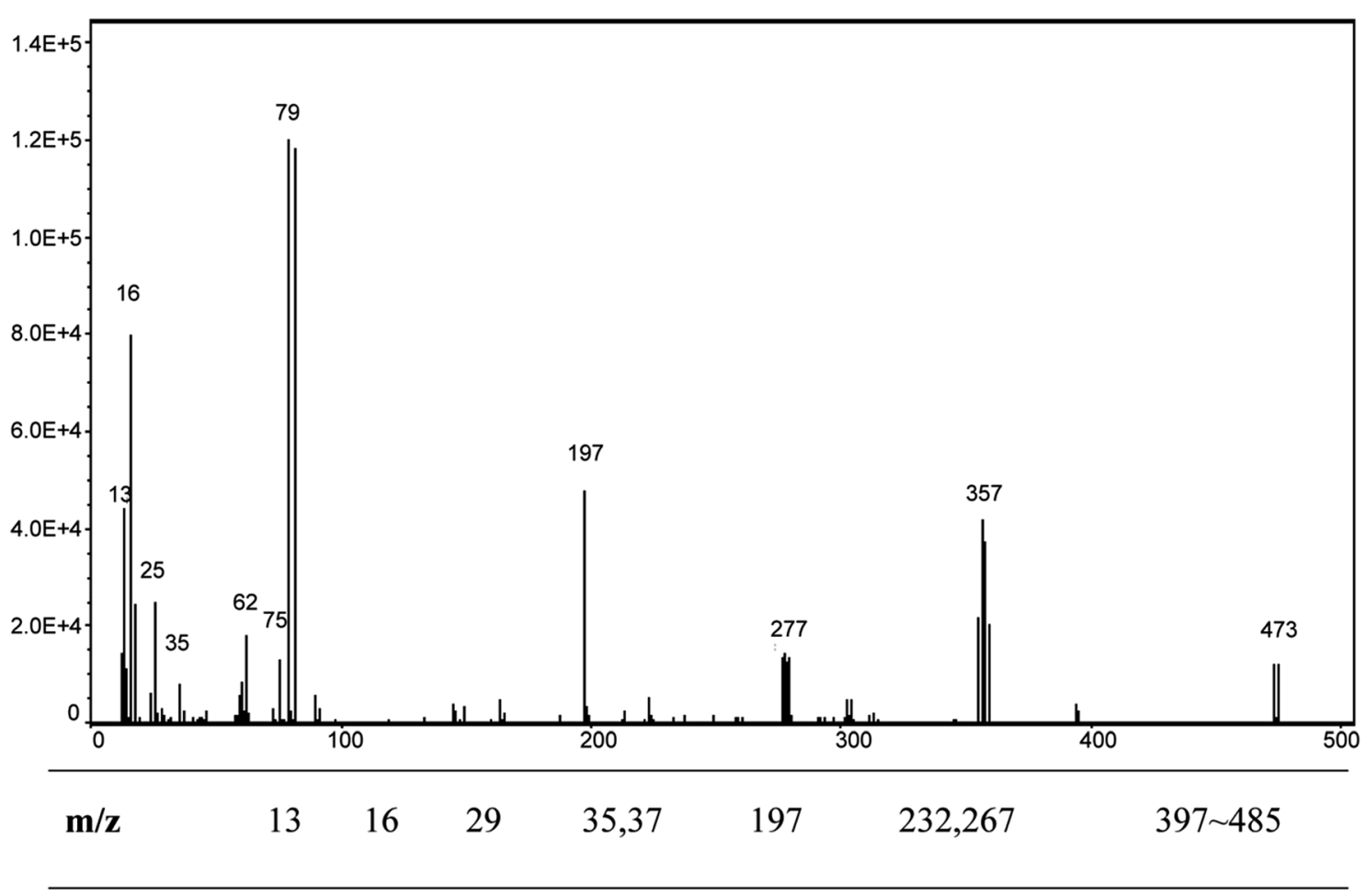

\section{Main Ions $\quad \mathrm{CH}^{-} \quad \mathrm{O}^{-} \quad \mathrm{C}_{2} \mathrm{H}_{5}^{+} \quad{ }^{35} \mathrm{Cl}^{-},{ }^{37} \mathrm{Cl}^{-} \quad \mathrm{Au}^{-} \quad \mathrm{Au}^{+} \mathrm{Cl}^{-}, \mathrm{AuCl}_{2}^{-} \quad \mathrm{C}_{\mathrm{n}} \mathrm{H}_{2 \mathrm{n}+2} \mathrm{P}\left(\mathrm{M}^{+}\right)$}

Fig. 6 ToF-SIMS spectrum and identification of the characteristic peaks of Au@P ${ }_{14,6,6,6} \mathrm{NTf}_{2} \mathrm{NPs}_{\text {. }}$ 


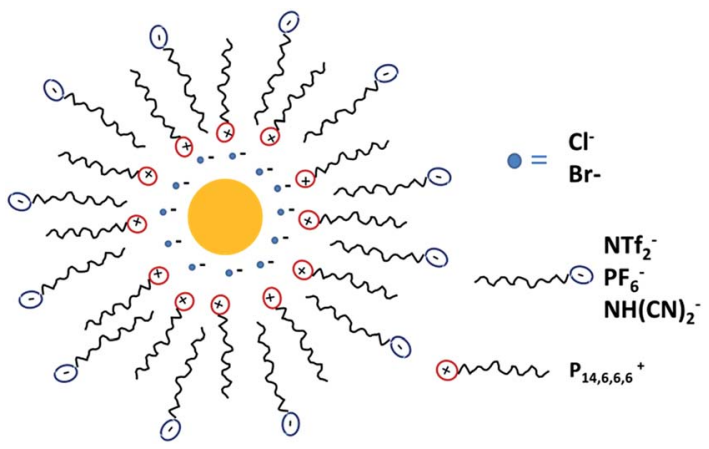

Fig. 7 Schematic illustration of the proposed stabilization mechanism of an Au@RTIL NP. Note the anion of RTIL may be absent in the surface layers.

surfaces, while the cation of the RTIL orderly arranges outside. This structure can also bring excellent colloidal stability. However, if the cations of RTIL are loosely packed in the outer layer, the stability of the Au NPs is relatively poor. The proposed surface layer structure of the as-prepared Au@RTIL NPs is shown in Fig. 7.

\section{Conclusions}

In summary, we successfully introduced hydrophobic RTILs into the BS method to synthesize Au NPs. The RTILs play dual roles as phase transfer agent and ligand which significantly simplify the synthetic system. The obtained Au@RTILs shows small size, narrow size distribution and outstanding colloidal stability. The Au@RTILs NPs can be easily re-dispersed into the toluene after being thoroughly dried. The size of the Au@RTIL NPs can be controlled simple by adjusting the molar ratio of RTIL to $\mathrm{AuCl}_{4}{ }^{-}$. The more RTIL added, the smaller Au NPs obtained. TGA, FTIR and ToF-SIMS characterization revealed that the adsorption of RTILs on Au surfaces, their interactions with $\mathrm{Au}$ surfaces and their degree of order on Au surfaces determine the stability of the obtained Au NPs. These findings disclose the mechanism of RTIL stabilization which will guide the RTILs assisted synthesis of high quality Au NPs in the future research. In our method, the utilization of TOAB was avoided. It is reported that the utilization of TOAB brings product with impurity of bromide that cannot be completed removed, ${ }^{35}$ while these impurities are toxic for further applications. Therefore, preparation of high quality $\mathrm{Au}$ NPs such as $\mathrm{Au} @ \mathrm{P}_{14,6,6,6} \mathrm{NTf}_{2}$ without $\mathrm{Br}$ will benefit their applications, especially in biology research. We believe that the excellent stability of Au NPs in organic medium and various function groups originated from different structures of RTIL will provide a novel platform for further functionalization and application of these Au@RTIL NPs.

\section{Acknowledgements}

This work is finically supported by Australia Research Council (DE120101788 and DE120100042) and University of South Australia (Foundation Fellow).

\section{Notes and references}

1 M. C. Daniel and D. Austruc, Chem. Rev., 2004, 104, 293.

2 S. E. Skrabalak, J. Y. Chen, Y. G. Sun, X. M. Lu, L. Au, C. M. Cobley and Y. N. Xia, Acc. Chem. Res., 2008, 41, 1587.

3 L. M. Liz-Marzán, Langmuir, 2005, 22, 32-41.

4 V. Zielasek, B. Jurgens, C. Schulz, J. Biener, M. M. Biener, A. V. Hamza and M. Baumer, Angew. Chem., Int. Ed., 2006, 45, 8241.

5 P. Herves, M. Perez-Lorenzo, L. M. Liz-Marzan, J. Dzubiella, Y. Lu and M. Ballauff, Chem. Soc. Rev., 2012, 41, 5577.

6 A. Ma, Y. Xie, J. Xu, H. Zeng and H. Xu, Chem. Commun., 2015, 51, 1469.

7 M. Mahyari, A. Shaabani and Y. Bide, RSC Adv., 2013, 3, 22509.

8 J. Zeng, Q. Zhang, J. Chen and Y. Xia, Nano Lett., 2009, 10, 30. 9 D. Liu, F. Zhou, C. Li, T. Zhang, H. Zhang, W. Cai and Y. Li, Angew. Chem., Int. Ed., 2015, 54, 9596.

10 Y. Ding and M. W. Chen, MRS Bull., 2009, 34, 569.

11 R. Bardhan, S. Lal, A. Joshi and N. J. Halas, Acc. Chem. Res., 2011, 44, 936.

12 M. De, P. S. Ghosh and V. M. Rotello, Adv. Mater., 2008, 20, 4225.

13 J. Turkevich, P. C. Stevenson and J. Hillier, Discuss. Faraday Soc., 1951, 11, 55.

14 M. Brust, M. Walker, D. Bethell, D. J. Schiffrin and R. Whyman, J. Chem. Soc., Chem. Commun., 1994, 801.

15 P. X. Zhao, N. Li and D. Astruc, Coord. Chem. Rev., 2013, 257, 638.

16 L. M. Liz-Marzan, Chem. Commun., 2013, 49, 16.

17 J. Zhou, D. A. Beattie, R. Sedev and J. Ralston, Langmuir, 2007, 23, 9170.

18 C. I. Müller and C. Lambert, Langmuir, 2011, 27, 5029.

19 X. T. Feng, Y. Zhang, J. J. Zou, X. W. Zhang and L. Wang, Mater. Lett., 2014, 118, 196.

20 L. L. Zhu, C. Zhang, C. C. Guo, X. L. Wang, P. C. Sun, D. S. Zhou, W. Chen and G. Xue, J. Phys. Chem. C, 2013, 117, 11399.

21 O. Zaluzhna, Y. Li, C. Zangmeister, T. C. Allison and Y. J. Tong, Chem. Commun., 2012, 48, 362.

22 Y. Li, O. Zaluzhna, C. D. Zangmeister, T. C. Allison and Y. J. Tong, J. Am. Chem. Soc., 2012, 134, 1990.

23 P. J. G. Goulet and R. B. Lennox, J. Am. Chem. Soc., 2010, 132, 9582.

24 M. Brust, J. Fink, D. Bethell, D. Schiffrin and C. Kiely, J. Chem. Soc., Chem. Commun., 1995, 1655.

25 M. J. Hostetler, S. J. Green, J. J. Stokes and R. W. Murray, J. Am. Chem. Soc., 1996, 118, 4212.

26 Y. J. Zhu, W. W. Wang, R. J. Qi and X. L. Hu, Angew. Chem., Int. Ed., 2004, 43, 1410.

27 L. A. Blanchard, D. Hancu, E. J. Beckman and J. F. Brennecke, Nature, 1999, 399, 28.

28 J. Yang, F. Kubota, Y. Baba, N. Kamiya and M. Goto, Solvent Extr. Res. Dev., Jpn., 2014, 21, 89.

29 A. Safavi and S. Zeinali, Colloids Surf., A, 2010, 362, 121. 
30 A. Safavi, S. Zeinali and M. Yazdani, Amino Acids, 2012, 43, 1323.

31 H. Zhang and H. Cui, Langmuir, 2009, 25, 2604.

32 R. J. Cornmell, C. L. Winder, G. J. Tiddy, R. Goodacre and G. Stephens, Green Chem., 2008, 10, 836.
33 C. K. Yee, R. Jordan, A. Ulman, H. White, A. King, M. Rafailovich and J. Sokolov, Langmuir, 1999, 15, 3486.

34 M. Dasog and R. W. Scott, Langmuir, 2007, 23, 3381.

35 C. A. Waters, A. J. Mills, K. A. Johnson and D. J. Schiffrin, J. Chem. Soc., Chem. Commun., 2003, 540. 